'Servicio de Medicina, Sección Nefrología, Hospital Naval A. Nef, Viña del Mar, Chile. 2Escuela de Medicina, Universidad de Valparaíso, Valparaíso, Chile. ${ }^{3}$ Servicio de Medicina, Sección Nefrología, Hospital Dr. Gustavo Fricke, Viña del Mar, Chile. ${ }^{4}$ Departamento de Endocrinología, Escuela de Medicina, Pontificia Universidad Catolica de Chile, Santiago, Chile. aBecado de SOCHED.

Recibido el 22 de abril de 2015, aceptado el 15 de junio de 2015.

Correspondencia a: Dr. Jorge Vega 5 Norte 1035, Viña del Mar, Chile.

Teléfono: 56322974237. jvegastieb@gmail.com

\section{Vasculitis de grandes vasos en paciente con vasculitis ANCA (+). Caso clínico}

\author{
JORGE VEGA ${ }^{1,2,3}$, FRANCISCO J. GUARDA, a
}

\section{Large vessel involvement in ANCA-associated vasculitis. Report of one case}

\begin{abstract}
Pauci-immune glomerulonephritis in systemic vasculitides usually have anti-neuthrophil cytoplasmic antibodies (ANCA). However, vasculitides oflarge vessels such as Takayasu's and giant cell (temporal) arteritis do not. Exceptionally ANCA(+) small vessel vasculitides are associated with large vessel vasculitis. It may be a coincidence or both vasculitides have a common pathogenesis. We report a 30 years old woman on hemodialysis due to a chronic glomerulonephritis ANCA(+) diagnosed nine years ago. Eight years later, she presented with an aortitis with severe stenosis of distal aorta and vasculitis of left subclavian artery. She was treated with adrenal steroids and cyclophosphamide. During the ensuing five years she has been stable and without signs of reactivation of the disease.

(Rev Med Chile 2015; 143: 1206-1209)
\end{abstract}

Key words: Antibodies anti-neuthrophil cytoplasmic; Aortitis; Takayasu arteritis.

\footnotetext{
L
} as vasculitis de vasos pequeños, que son la poliangeitis microscópica, granulomatosis con poliangeitis (Wegener), granulomatosis eosinofílica con poliangeitis (Churg-Strauss) y glomerulonefritis crescéntica idiopática pauciinmune, frecuentemente tienen anticuerpos anticitoplasma de los neutrófilos (ANCA) detectables al momento del diagnóstico. Las vasculitis de vasos grandes, en cambio, no los tienen ${ }^{1,2}$. El compromiso de vasos grandes en las vasculitis de vasos pequeños $\mathrm{ANCA}(+)$ es raro $^{1-18}$. Algunos autores consideran que es una coincidencia de dos patologías, otros creen que no lo es, sino que existe una patogénesis común ${ }^{1,4,12}$.

Comunicamos el caso de una paciente que presentó una vasculitis de vasos pequeños $\mathrm{ANCA}(+)$ con compromiso renal y años después, una aortitis con severa estenosis de la aorta abdominal.

\section{Caso clínico}

Mujer de 30 años, hospitalizada el 2002 por poliartritis y nefropatía (proteinuria, hematuria, creatinina $2,0 \mathrm{mg} / \mathrm{dl}$, clearance de creatinina $49 \mathrm{ml} / \mathrm{min} / 1,73 \mathrm{~m}^{2}$ ). Se le indicó prednisona, la cual abandonó a los 4 meses por aparición de facies cushingoide, así también sus controles médicos.

Un año más tarde (2003) consultó por hipertensión arterial prescribiéndose carvedilol. Existía insuficiencia renal (creatinina 1,7 mg/dl, clearance de creatinina $54 \mathrm{ml} / \mathrm{min} / 1,73 \mathrm{~m}^{2}$ ), proteinuria $400 \mathrm{mg} / 24 \mathrm{~h}$, anemia, VHS elevada y $\operatorname{ANCA}(+)$ (patrón perinuclear). Se hospitalizó para efectuar biopsia renal (BpR) por sospecha de glomerulonefritis crescéntica pero la enferma se fugó horas después y la BpR no se realizó. Reapareció 3 meses después, hipertensa $(160 / 100 \mathrm{mmHg})$, su función renal estaba en rangos similares, había hematuria microscópica, leucocituria, proteinuria $(1.890 \mathrm{mg} /$ día) y $\mathrm{ANCA}(+)$ a dilución 1/640 (inmunofluorescencia indirecta). Como no aceptó efectuarse una $\mathrm{BpR}$ teniendo el diagnóstico clínico de glomerulonefritis ANCA $(+)$ se indicó ciclofosfamida $50 \mathrm{mg} /$ día oral, deflazacort $30 \mathrm{mg} /$ día (para evitar los efectos cosméticos de otros corticoides) y telmisartán $40 \mathrm{mg}$ /día. Al final de ese año su función renal había mejorado (clearance de creatinina 62 
$\mathrm{ml} / \mathrm{min} / 1,73 \mathrm{~m}^{2}$ ), no había proteinuria (uso conjunto de lisinopril y telmisartan) y recibía $50 \mathrm{mg}$ de ciclofosfamida y 7,5 mg de deflazacort.

Al tercer año de su enfermedad (2004) presentó amenorrea (toxicidad por ciclofosfamida) y reactivación de su vasculitis con empeoramiento marcado de la función renal (creatinina: $4,0 \mathrm{mg} /$ $\mathrm{dl}$; clearance de creatinina: $\left.16 \mathrm{ml} / \mathrm{min} / 1,73 \mathrm{~m}^{2}\right)$, reaparición de la proteinuria $(+++)$, hematuria microscópica, elevación de VHS $(70 \mathrm{~mm} / \mathrm{h})$ y ANCA (1/1.280 por inmunofluorescencia indirecta). No se determinó ELISA para PR3 ni MPO. Se prescribió deflazacort $60 \mathrm{mg} /$ día y ciclofosfamida $150 \mathrm{mg} /$ día logrando reducir la severidad de su insuficiencia renal. Al final de ese año el clearance de creatinina fue: $65 \mathrm{ml} / \mathrm{min} / 1,73 \mathrm{~m}^{2}$, proteinuria: $650 \mathrm{mg} / 24$ h y no había hematuria.

Al cuarto año (2005), se cambió la ciclofosfamida por azatioprina, manteniendo dosis bajas de deflazacort, telmisartan y lisinopril. Al final del año la creatinina era $1,5 \mathrm{mg} / \mathrm{dl}$, clearance de creatinina: $68 \mathrm{ml} / \mathrm{min} / 1,73 \mathrm{~m}^{2}$ y proteinuria: $480 \mathrm{mg} / 24 \mathrm{~h}$.

$\mathrm{Al}$ quinto año presentó herpes zoster intercostal. Al año siguiente estaba normotensa, su función renal permanecía estable (clearance de creatinina: $64 \mathrm{ml} / \mathrm{min} / 1,73 \mathrm{~m}^{2}$ ) y la proteinuria: $709 \mathrm{mg} / 24 \mathrm{~h}$. Al séptimo año no acudió a controles.

Reapareció al octavo año por hematuria macroscópica y sinusitis aguda. Si bien afirmó mantener la terapia indicada, la función renal se había deteriorado (creatinina: 4,1 mg/dl, uremia: $122 \mathrm{mg} / \mathrm{dl}$ ), examen de orina: cilindros hemáticos, céreos y cuerpos ovales grasos. Se prescribió antibióticos y se citó a control en 1 semana. Reapareció 6 meses más tarde con notorio compromiso del estado general, falla renal (creatinina: $7,4 \mathrm{mg} / \mathrm{dl}$, clearance de creatinina: $12 \mathrm{ml} / \mathrm{min} / 1,73 \mathrm{~m}^{2}$; uremia: $185 \mathrm{mg} / \mathrm{dl}$ ) y proteinuria: $4.534 \mathrm{mg} / 24 \mathrm{~h}$ ). La serología para virus B, C y VIH fue negativa. Se instaló un catéter tunelizado, inició hemodiálisis crónica y se construyó una fístula arterio-venosa (FAV). Posterior a ello evolucionó en buenas condiciones.

Seis meses después, una semana después de comenzar a utilizar la FAV para diálisis, acudió a control en mal estado general, disneica e intensamente febril y anémica. Había frotes pericárdicos, derrame pleural bilateral, leucocitosis $\left(12.200 \mathrm{~mm}^{3}\right)$, VHS: $140 \mathrm{~mm} / \mathrm{h}$ y PCR: $354 \mathrm{mg} / \mathrm{L}$ (VN: < 9). Se planteó como hipótesis diagnósticas: sepsis por catéter, endocarditis infecciosa, infección oportunista, reactivación de la vasculitis y diálisis insuficiente. Se tomó hemocultivos en sangre periférica y por catéter (retirándolo inmediatamente), se administró antibióticos, se dializó diariamente y se transfundió glóbulos rojos. En los días siguientes su estado general mejoró, la fiebre cayó paulatinamente y desaparecieron los frotes y derrames pleurales. Los hemocultivos y urocultivo fueron negativos, el ecocardiograma mostró un derrame pericárdico (no habían vegetaciones), los ANA fueron negativos, complemento C'3 y C'4: normal y los ANCA por inmunofluorescencia y ELISA fueron negativos. Estando la paciente en condiciones aceptables exigió el alta, aunque no se conociera la etiología de su cuadro febril y grave descompensación.

Días más tarde, reapareció la fiebre. El scanner de tórax- abdomen-pelvis efectuado buscando la causa de la fiebre, mostró estenosis acentuada de la aorta abdominal (bajo arterias renales indemnes) con paso filiforme hacia las arterias iliacas primitivas, que presentaban dilatación post-estenótica marcada (Figura 1). Existía además un tejido inflamatorio periaórtico y alrededor de la arteria subclavia izquierda que tenía una estenosis proximal. Los riñones eran pequeños. El radiólogo sospechó una arteritis de Takayasu. Se reinició la terapia con corticoides y ciclofosfamida.

En los 5 años siguientes ha estado en buenas condiciones y no han aparecido signos de reactivación de su enfermedad. En este período, a pesar de no tener signos isquémicos en sus miembros inferiores y superiores (Figura 1), se efectuó un bypass aorto-ilíaco, con miras de someterla a un trasplante renal. Durante la intervención se resecó el segmento aórtico distal que mostró fibrosis de la adventicia con fusión a la muscular (que estaba atrófica) e hiperplasia de la íntima, compatibles con secuelas de una aortitis. No se encontraron granulomas, necrosis ni células gigantes.

\section{Discusión}

Esta paciente debutó con una glomerulonefritis $\mathrm{ANCA}(+)$ y compromiso articular a los 30 años. La terapia inmunosupresora logró estabilizar su nefropatía. En los años posteriores tuvo algunas complicaciones usuales de esta terapia y reactivación de su vasculitis, respondiendo satisfactoriamente. Ocho años después, en relación a una infección respiratoria y probablemente a abandono del tratamiento, sufrió reactivación de 

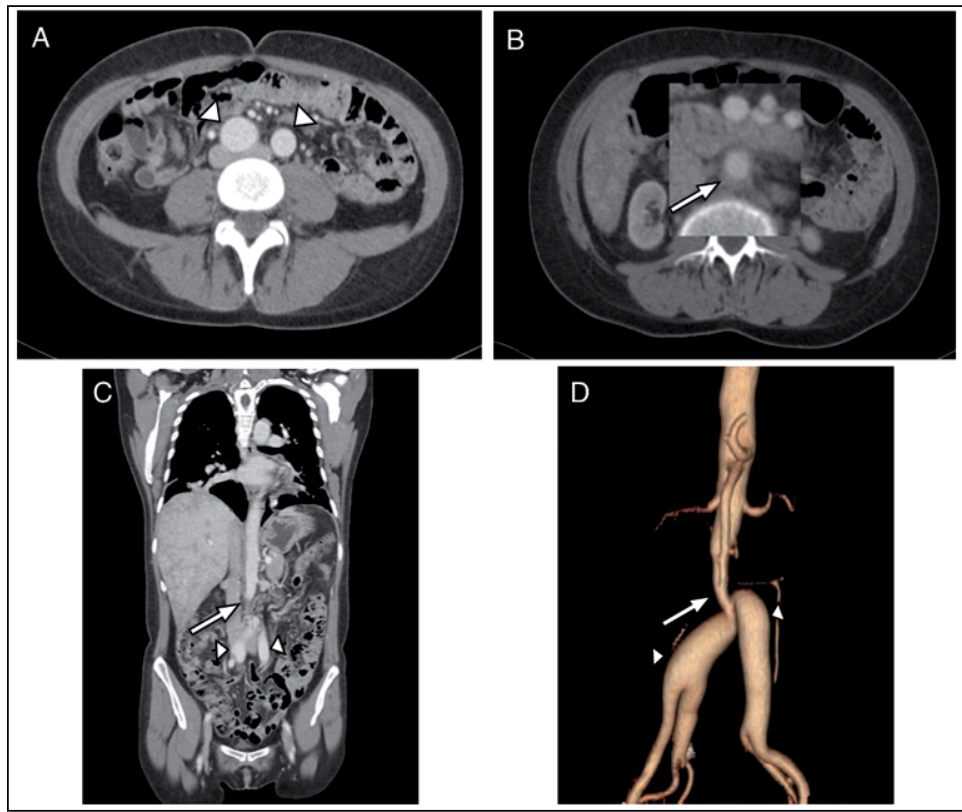

Figura 1. Imágenes de aorta y arterias ilíacas. A) Corte transversal a nivel umbilical. Se observan lúmenes de arterias iliacas primitivas muy dilatados (cabezas de flechas blancas); B) Corte transversal de abdomen a la altura del riñón derecho (atrófico). Se observa un halo peri-aórtico propio de una aortitis (flecha blanca); C) Corte coronal de tórax-abdomen-pelvis. Se observa estrechamiento severo de la aorta abdominal distal (flecha blanca) y dilatación de arterias iliacas primitivas (cabezas de flechas blancas); D) Reconstrucción de aorta abdominal y arterias iliacas. Se observa estenosis filiforme de aorta distal (flecha blanca) y dilatación post-estenótica de arterias iliacas (cabezas de flechas blancas). su vasculitis con pérdida definitiva de la función renal. Seis meses después de ingresar a hemodiálisis presentó un severo cuadro inflamatorio descubriéndose en una tomografía la presencia de vasculitis de la arteria subclavia y aortitis, que fue comprobada histológicamente más tarde.

En los casos publicados de asociación de vasculitis en vasos pequeños y grandes, la ocurrencia ha sido simultánea en la mayoría de los casos. Cuando fue metacrónica, el intervalo varió entre 6 meses y 22 años $^{5-7,9,14}$. En esta paciente fue 9 años.

En las vasculitis de grandes vasos deben descartarse: sífilis, tuberculosis, lupus eritematoso, enfermedad de Behcet, espondiloatropatías, policondritis recurrente, síndrome de Cogan, artritis reumatoide, enfermedad de Still, enfermedad de Buerger y polimiositis ${ }^{1,2}$. Ellas se descartaron razonablemente en esta paciente. El diagnóstico diferencial fundamental debe hacerse con arteritis de células gigantes(temporal) y enfermedad de Takayasu. La primera es propia de sujetos mayores y la segunda de mujeres jóvenes, como esta paciente, sin embargo, estas vasculitis no se asocian a la presencia de ANCA. Chirinos y cols. propusieron una variedad distinta de vasculitis: de vasos grandes asociada a $\mathrm{ANCA}^{2}$. Este tipo de vasculitis puede originar periarteritis (periaortitis), aortitis estenosante (como este caso), dilatación, aneu- rismas, oclusión, disección y ruptura aórtica ${ }^{1-3}$.

En los casos comunicados, además de la existencia de glomerulonefritis crescéntica ANCA(+) y aortitis (toráxica o abdominal), se ha descrito compromiso de válvula aórtica, tronco braquiocefálico, carótidas, subclavias, axilar, ilíacas comunes, pulmonares y ostium de las arterias coronarias $^{1,4,10-18}$.

Si bien el mecanismo patogénico de este tipo de aortitis no es conocido, se ha propuesto que los ANCA originarían una vasculitis de vaso vasorum en la adventicia y que la inflamación se extendería a la media e íntima ${ }^{2}$. Otros autores han planteado que una vasculitis de las arterias retroperitoneales (periaortitis) invadiría la pared de la aorta ${ }^{3,7}$. Las biopsias efectuadas durante la reparación de aneurismas, revascularización o necropsias han revelado vasculitis de vasa vasorum y arterias retroperitoneales, inflamación, granulomas, rotura de las fibras elásticas de la media, necrosis fibrinoide y vasculitis necrotizante granulomatosa ${ }^{2-9,19}$. Si bien estos hallazgos son comunes con las vasculitis de grandes vasos clásicas, la necrosis parece ser un hecho distintivo las vasculitis $\operatorname{ANCA}(+)^{3}$.

No existe una terapia establecida para este tipo de vasculitis. Se ha utilizado la combinación de ciclofosfamida y esteroides y en casos refractarios, tocilizumab ${ }^{2,3,17}$. El pronóstico es similar a las 
vasculitis de vasos pequeños $\mathrm{ANCA}(+)$ pero han ocurrido muertes por rotura y disección aórtica ${ }^{2}$.

En esta paciente la aparición metacrónica de una vasculitis de vasos pequeños $\mathrm{ANCA}(+)$ manifestada por una glomerulonefritis y de una vasculitis de vasos grandes diagnosticada 9 años después, plantea el interrogante si se trata de dos patologías diferentes o de la entidad propuesta por Chirinos y cols². El compromiso de la arteria subclavia, la negatividad de los ANCA al momento del diagnóstico de la aortitis en el acmé del cuadro inflamatorio, apoya la primera posibilidad. Otra posibilidad planteable es que desde el principio su nefropatía fuera por enfermedad de Takayasu, sin embargo, la presencia de ANCA, indemnidad de las arterias renales y presentación como síndrome nefrítico no son compatibles. Afortunadamente la terapia implementada (ciclofosfamida+esteroides) ha logrado mantener en remisión clínica ambos trastornos permitiendo que la paciente pueda ser trasplantada de riñón.

El objeto de la presentación de este caso es advertir que las vasculitis de vasos pequeños $\mathrm{ANCA}(+)$ pueden asociarse ocasionalmente a compromiso de vasos grandes.

\section{Referencias}

1. Nakabayashi K, Kamiya Y, Nagasawa T. Aortitis síndrome associated with perinuclear antineutrophil cytoplasmic antibody: report of three cases. Int J Cardiol 2000; 75 Suppl 1: S89-S94.

2. Chirinos JA, Tamariz LJ, Lopes G, Del Carpio F, Zhang $\mathrm{X}$, Milikowski C, et al. Large vessel involvement in ANCA-associated vasculitides: report of a case and review of the literature. Clin Rheumatol 2004; 23: 152-9.

3. Carels T, Verbeken E, Blockmans D. p-ANCA-associated periaortitis with histological proof of Wegener's granulomatosis. case report. Clin Rheumatol 2005; 24: 83-6.

4. Baker-Le Pain JC, Farmer-Boatwright MK, Dooley MA. Concurrent Takayasu's arteritis and antineutrophil cytoplasmic antibody-related glomerulonephritis related to use of propylthiouracil. J Rheumatol 2009; 36: 1552-4.

5. Unlü C, Willems M, Ten Berge IJ, Legemate DA. Aortitis with aneurysm formation as a rare complication of Wegener's granulomatosis. J Vasc Surg 2011; 54: 1485-7.

6. Ohta N, Waki T, Fukase S, Susuki Y, Kurakami K, Aoyagi $\mathrm{M}$, et al. Aortic aneurysm rupture as a rare complication of granulomatosis with polyangiitis: a case report. J Med Case Rep 2013; 7: 202.

7. Blockmans D, Baeyens H, Van Loon R, Lauwers G,
Bobbaers H. Periaortitis and aortic dissection due to Wegener's granulomatosis. Clin Rheumatol 2000; 19: 161-4.

8. Fink AM, Miles KA, Wraight EP. Indium-111 labelled leucocyte uptake in aortitis. Clin Radiol 1994; 49: 863-6.

9. Sieber SC, Cuello B, Gelfman NA, Garfinkel HB. Pulmonay capillaritis and glomerulonephritis in an antineutrophil cytoplasmatic antibody-positive patient with prior granulomatosus aortitis. Arch Pathol Lab Med 1990; 114: 1223-6.

10. Hellmann DB, Hardy K, Lindenfeld S, Ring E. Takayasu's arteritis associated with crescentic glomerulonephritis. Arthritis Rheum 1987; 30: 451-4.

11. Morshuis WJ, Zeebregts CJ, Haanen HC, Elbers JR, Ernst JM, Vermeulen FE. Aortitis, aortic valve incompetence, and left coronary ostium stenosis in a patient with C-ANCA-associated necrotizing vasculitis. Thorac Cardiovasc Surg 1997; 45: 97-9.

12. Logar D, Rozman B, Vizjak A, Ferluga D, Mulder AHL, Kallenberg CGM. Arteritis of both carotid arteries in a patient with focal, crescentic glomerulonephritis and anti-neutrophil cytoplasmic autoantibodies. Rheumatology 1994; 33: 167-9.

13. Sakemi T, Tomiyoshi Y, Yano H, Ikeda Y, Matsuo Y, Kudo S. Retroperitoneal fibrosis with perinuclear antineutrophil cytoplasmic antibodies and a longitudinally extended periaortic soft-tissue structure on CT. Nephron 1998; 78: 218-20.

14. de RS, Serratrice J, Granel B, Disdier P, Bartoli JM, Pache X, et al. Periaortitis heralding Wegener's granulomatosis. J Rheumatol 2002; 29: 392-4.

15. Wiatr E, Dobkowski P, Opoka L, Drygalska-Pozoronska A, Orlowski T, et al. Wegener's granulomatosis overlapped with Takayasu arteritis complicated by thrombosis of internal jugular vein. Pneumonol Alergol Pol 2003; 71: 440-8.

16. Takenaka K, Ohba T, Suhara K, Sato Y, Nagasaka K. Successful treatment of refractory aortitis in antineutrophil cytoplasmic antibody-associated vasculitis using tocilizumab. Clin Rheumotal 2014; 33: 287-9.

17. Murakami S, Saito H, Ohe M, Kondo T, Oshita F, Yamada K. Periaortitis associated with anti-neutrophil cytoplasmic antibodies induced by bevacizumab combination therapy. Intern Med 2013; 52: 589-91.

18. Kim BK, Park SY, Choi CB, Kim TH, Jun JB,Jang SM, et al. A case of microscopic polyangiitis associated with aortic valve insufficiency. Rheumatol Int 2013; 33: 10558.

19. Vaglio A, Corradi D, Manenti L, Ferretti S, Garini G, Buzio C. Evidence of autoimmunity in chronic periaortitis: a prospective study. Am J Med 2003; 114: 454-62. 